\title{
Condensateurs synchrones
}

De nombreuses communications ont été publiées ces temps derniers sur la nouvelle tarification de l'énergie électrique, notamment en tenant compte de la puissance réactive (ou déwattée), et sur les divers systèmes préconisés pour relever le cosinus des installations existanles.

Les moyens actuellement employés pour améliorer le facteur de puissance d'une installation électrique sont les candensateurs statiques, les moteurs synchrone's surexcités designés couramment par analogie sous le nom de "Condensateurs synchrones" et les Avanceurs de phase (1).

Les condensateurs statiques sont surtout employés dans les petites installations utilisant seulement une puissance de quelques kilowatts. Ils présentent l'avantage d'une installation facile ct d'un entretien à pe'u près nul. Lorsqu'il s'agit d'obtenir une puissance réactive ou magnétisante dépassant $20 \mathrm{kVA}$ les condensateurs statiques, dont l'encombrement et le prix d'achat deviennent très impartants, sont avantageusement remplacés par les condensateurs synchrones.

L'emploi des condensateurs synchrones eșt la solution la plus économique pour relever le cos des instatlations de moyenne ou grande puissance, utilisant prineipalement des moteurs d'induction de puissance assi: faible, qu'ils s'agisse d'industriels auxquels un cos minimum est imposé ou de secteurs de distribrtion qui ve'ulent améliorer le fonctionnement de leur réseau.

On sail en effet que le moteur synchrone est susceptible de fournir en même lemps qüun couple moleur sur son arbre une puissance réactive sur le réseau qui l'alimente et de pouvoir remplir ainsi par simple variation de son excitation soit le rôle d'une self, soit le rôle d'une capacité.

Yous n'envisagerons principalement dans cet article que son application comme condensateur. Nous supposerons donc que le couple moteur qu'on lui demande est nul et dans ces conditions la puissance wallíe absorbée se réduit à la valeur des pertes dans la machine, soit environ de 1 à 6 ó de la puissance nominale.

It y a cependant lieu de noter que son emploi comme self est quèlquefois très intéressant, notamment au moment de la mise sous tension d'une ligne dont la capacilé est importante.

\section{Fonctionatement D'UN MotelR SyNChrone}

Nous rappellerons brièvement le principe du fonctionnement d'un moteur synchrone accroché sur un réseau et tournant à vide.

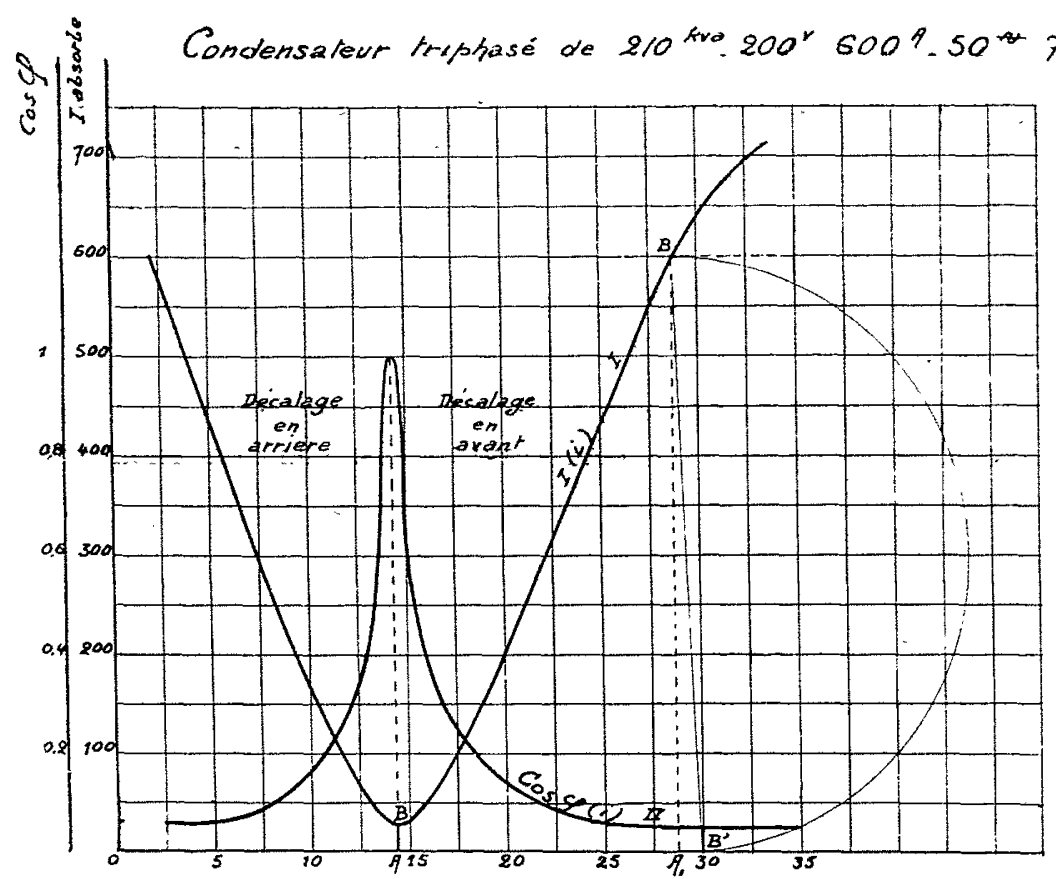

Fig. 1. - Condensateur triphasé $210 \mathrm{kVA}, 200 \mathrm{~V}, 600 \mathrm{~A}, 50 \mathrm{pps}$, $750 \mathrm{t} / \mathrm{m}$. Courbe 1 : intensité absorbée en fonction de l'excitation. Courbe 2 : facteur de puissance.

Le moteur étant à sa vitesse de synchronisme alimenté à tension constante U si l'on fait croître le courant d'excitation $i$, l'intensité absorbée I part d'une valeur sensiblement égale au courant de court-circuit qu'aurait la même machine fonctionnant en alter-

(1) Les avanceurs de phase sont uniquement utilisés pour améliorer le facteur de puissance des moteurs asynchrones avec lesquels ils sont couplés.

Article published by SHF and available at http://wWw.sht-lhb.org nateur avec l'excitation correspondant à une f.e. m. égale à $\mathrm{U}$, décroît, passe par un minimum, puis croît ensuite.

Ces variations sont représentées sur la figure 1 , le moteur tournant à charge nulle (caractéristique en V).

Le décalage du courant par rapport à la tension diminue tout d'abord (décalage en arrière), devient nul pour un minimum, puis augmente (décalage en avant). Voir courbe 2.

L'intensité déwaltéc correspondant à une excitation déterminée soblient d'une facon assez cxacte comme il est indiqué dans la figure 1 (triangle $\Lambda_{1} B_{1} B^{\prime}$ ) Le vecteur $\Lambda_{1} B^{\prime}$ correspond à l'intensité minimum absorbée $A \mathrm{~B}$.

La puissance réactive d'un moteur synchrone triphasé s'obtient immédiatement par le produit $V \overline{3} U \times B^{\prime} B_{1}$.

\section{CONSIDÉRATIONS THÉORIQUES SUR L'EMPLOI DES CONDENSATEURS SYNCHRONES}

L'emploi d'un moteur synchrone pour relever le facteur de puissance se déduit immédiatement des considérations précédentes.

Nous examinerons tout d'abord le cas le plus général d'une puissance vraie installée $P_{w}$, correspondant à une puissance apparente $\mathrm{P}_{0}$ dont l'intensité $\mathrm{I}_{0}$ est décalée d'un angle $\varphi_{0}$ sur la tension $U$, pour laquelle on désire relever le facteur de puissance jusqu'à une valeur cos o par l'adjonction d'un condensateur synchrone dont il y a lieu de déterminer la puissance déwattée $P_{d w}$.

C'est le cas d'une installation où la puissance wattée maximum (en $\mathrm{kw}$ ou en $\mathrm{Ch}$ ) reste constante, fixée par la police d'abonnement et dans laquelle le secteur qui fournit l'énergie impose un facteur de puissance minimum ou bien a établi un système de tarification de la puissance réactive.

Les relations suivantes nous donnent immédiatement :

$$
\begin{gathered}
\mathrm{P}_{\mathrm{w}}=\mathrm{P}_{0} \times \cos 5_{0} \\
\mathrm{I}_{0}=\frac{\mathrm{P}_{0} \times 1000}{\sqrt{3} \mathrm{U}}
\end{gathered}
$$

en courants alternatifs triphasés avec $\mathrm{P}_{0}$ exprimé en $\mathrm{kVA}$. 
Un relèvement du facteur de puissance de 0,60 à 0,9 par exemple nous donne $\frac{P_{d w}}{P_{0}}=0,51$ c'est-à-dire que pour une puissance installée $\mathrm{P}_{0}$ de $100 \mathrm{kVA}$, la puissance réactive nécessaire est $\mathrm{P}_{\mathrm{dw}}=51 \mathrm{kVA}$.

La nouvelle valeur du courant I se déduit immédiatement.

Les considérations précédentes permettent de résoudre un problème intéressant pour les secteurs ayant des lignes ou des machines au maximum de charge en ampères avec un faible cosinus et qui veulent déterminer la puissance supplémenlaire qu'ils pourront distribuer en plus par l'amélioration du facteur de puissance de leur réseau, le courant total distribué restant constant.

Le diagramme de la figure 5 donne la valeur du courant déwatté en avant $I_{d w}$ qui permet de fournir un courant supplémentaire $i$ décalé de $\tau_{1}$ sur la tension tout en maintenant le courant total distribué à la valeur initiale. Il est facile de déterminer les valeurs limites de $i$ pour un angle $i_{1}$ et une intensité $I_{d w}$ donnée.

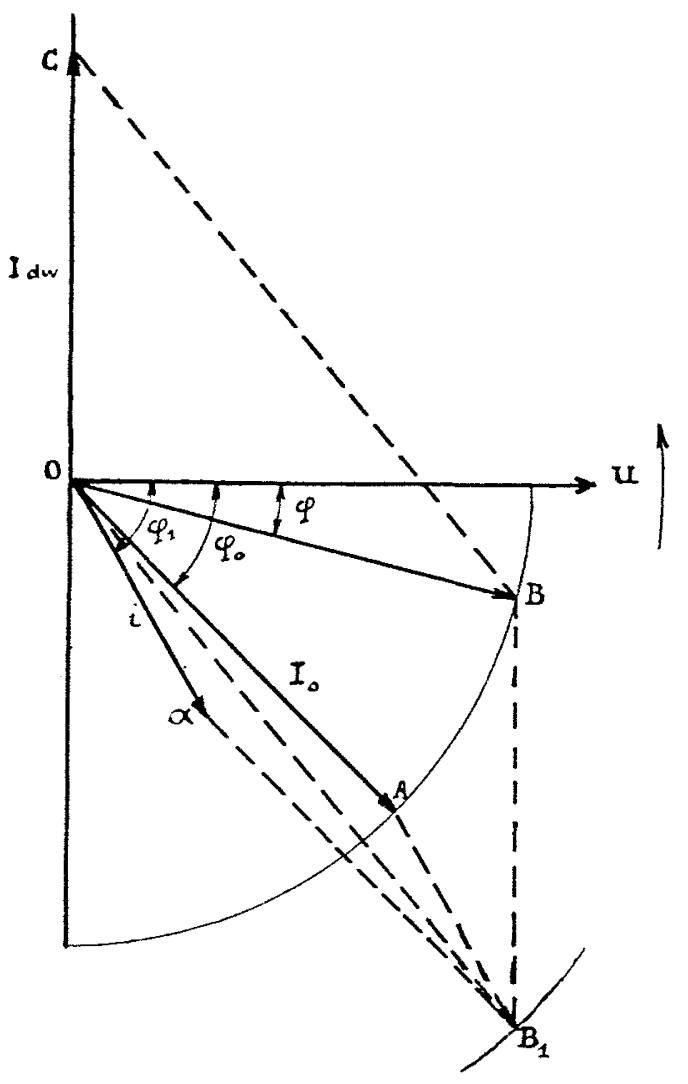

Fig. 5.

Considérations pratiques sur l'EMPLOI des CONDENŚAteurs SYNCHRONES

Les industriels qui s'étaient fort peu souciés jusqu'ici du cosinus de leur installation ont envisagé, selon les cas, le remplacement d'un ou plusieurs moteurs asynchrones par des moteurs synchrones, ou même par l'adjonction pure et simple d'un condensateur synchrone à l'installation primitive.

Nous avons indiqué précédemment les raisons qui justifient l'emploi du condensateur synchrone pour améliorer le facteur de puissance.

Il n'y a pas d'arguments très sérieux contre l'emploi de cette machine qui tendra d'ailleurs à être de plus en plus adoptée dans la pratique courante lorsque les exploitants auront pris contact avec elle.

L'installation d'un condensateur synchrone est identique à celle d'un moteur synchrone ou d'un alternateur. L'accrochage du condensateur sur le réseau nécessite les mèmes précautions :

10 Réaliser l'égalité cntre la force contre-électromotrice du moteur et la tension du réseau en réglant l'excitation à une valcur convenable.

$2^{\circ}$ Obtenir la concordance des phases en agissant légèrement sur la vitesse.

Le condensateur synchrone est amené à la vitesse de synchronisme soit au moyen d'un moteur auxiliaire, soit en le faisant fonctionner comme un moteur asynchrone pendant la période de démarrage.

\section{Démarrage par Moteur auxiliatre}

Un reproche sérieux fait au moteur synchrone est la faiblesse du couple de démarrage et du couple d'accrochage dans le démarrage en asynchrone.

C'est pour cette raison que l'on préfère souvent employer un moteur auxiliaire de démarrage monté en bout d'arbre du moteur synchrone, malgré la possibilité du démarrage en asynchrone.

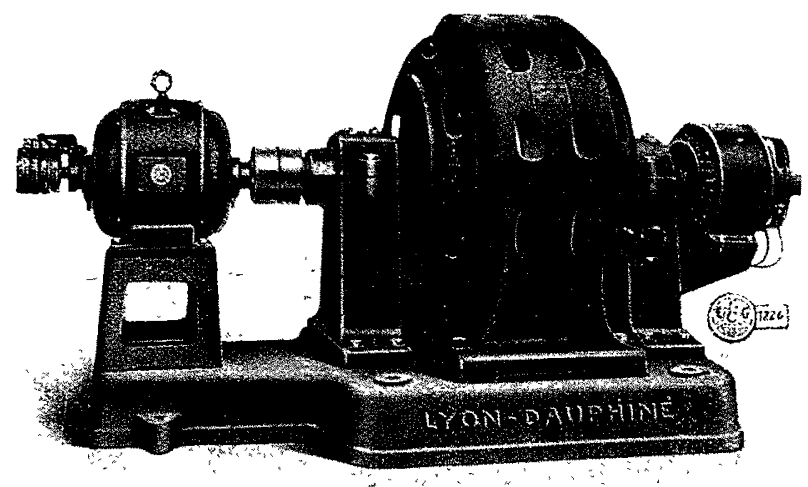

Fig. 6. - Condensateur synchrone de $210 \mathrm{kVA}, 200 \mathrm{~V}, 600 \mathrm{~A}$, $50 \mathrm{pps}, 750 \mathrm{t} / \mathrm{m}$, avec moteur auxiliaire de démarrage.

Les figures 6 et 9 représentent des condensateurs synchrones de $210 \mathrm{kVA}$ et $1200 \mathrm{kVA}$ prévus avec démarrage par moteur auxiliaire. La puissance de ce moteur auxiliaire est environ 5 à $6 \%$ de la puissance du moteur synchrone. La vitesse peut être égale ou supérieure à celle de ce dernier; dans ce dernier cas son rhéostat de démarrage doit être prévu assez largement pour servir de rhéostat de glissement et l'accrochage du condensateur synchrone sur un réseau ne présente alors aucune difficulté et s'effectue comme le couplage d'un alternateur dans la marche en parallèle.

Ce procédé très commode nécessite seulement une légère dépense supplémentaire.

\section{DÉMARRAGE EN ASYNCIIRONE}

Un condensateur synchrone dont le stator est alinenté sous une tension donnée, le circuit d'excitation étant ouvert ou fermé sur une résistance fonctionne comme un moteur asynchrone à cage d'écureuil. En donnant aux circuits amortisseurs formant cage. d'écureuil, une résistance appropricé, il est possible d'obtenir un couple de démarrage égal à 20 à $30 \%$ du couple normal du moteur synchrone, tout en n'absorbant sur le réseau qu'un à coup de courant égal à l'intensité de pleine charge.

En général la tension de démarrage est comprise entre 35 et $45 \%$ de la tension normale et il. est préférable d'exciter légèrement le moteur avant d'appliquer la tension totale de la ligne, 
Le magnélisme rémanant des pôles ainsi que la différence de réactance synchrone antagoniste el transversale sont généralement sulfisants pour que le moteur s'accroche avec la polarité convenable des inducleurs.

En démarrant avec le circuit d'excitation ouvert, il se peut qu'au moment où la machine ayant atteint le synchronisme, la polarilé des inducteurs obtenue en fermant le circuit d'excitation ne coincide pas avec celle induite par le champ alternatif à ce moment, lc moteur " glissera d'un pôle ", c'est-à-dire que le rotor se décalera dans l'espace d'un $1 / 2$ pas polaire de manière que les pôles inducleurs se trouvent vis-à-vis des pôles de sens contraire du champ tournant statorique.

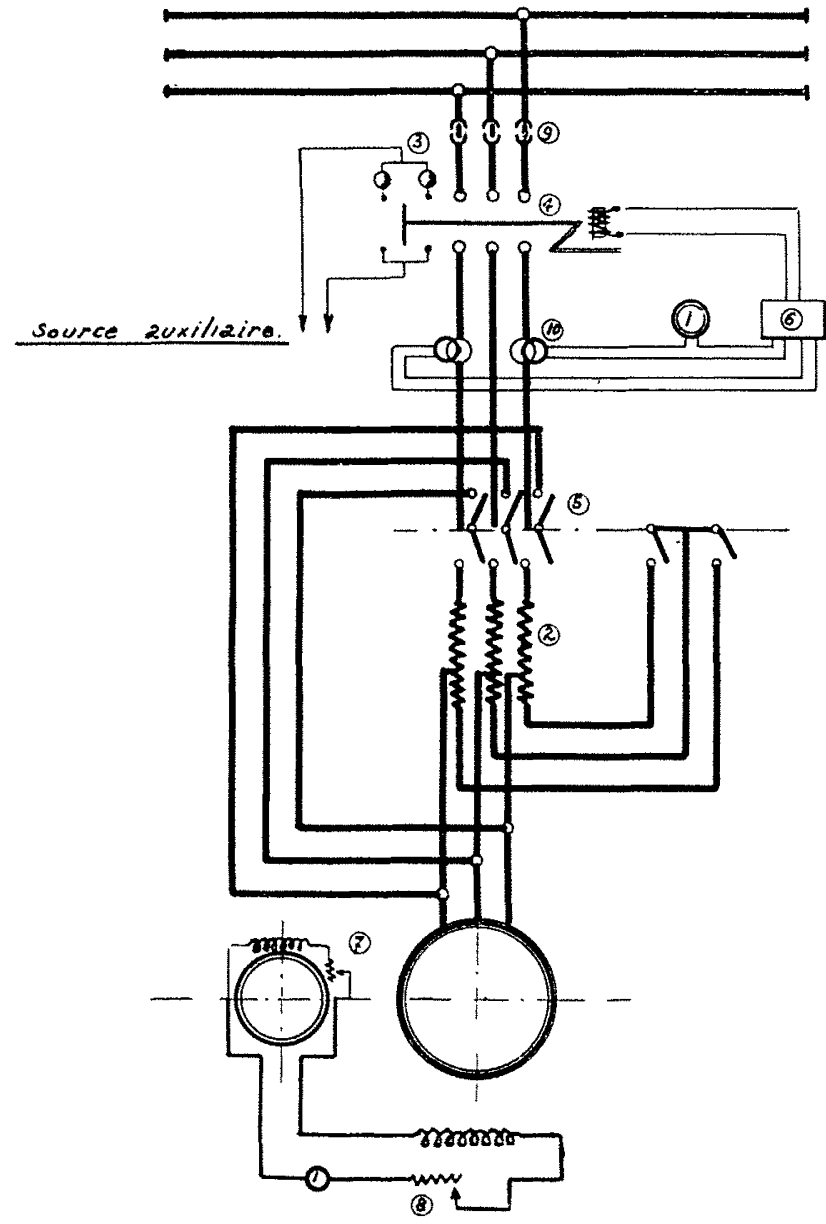

Fig. 7. - Démarrage d'un condensateur synchrone HT au moyen d'un auto-transformateur de démarrage.

Afin d'éviter l'à coup qui en résulte, il est préférable de fermer, avant la mise en marche, le circuit d'excitation sur une résistance (en général la résistance maximum du rhéostat de champ suffit). Dans ces conditions, malgré un affaiblissement du couple de démarrage, on réalisera une sorte d'autosynchronisation dès que la vitesse du rotor sera sufifsante pour permettre l'amorçage de l'excitatrice.

Notons qu'il peut arriver dans ce cas que le moteur conserve une vitesse voisine des $2 / 3$ de sa vitesse normale; cela est dû au fait que les inducteurs étant court-circuités sur une résistance insuffisante, forment un enroulement monophasé dont le champ est prépondérant par rapport à celui de forme polyphasée dû aux courants de Foucault induits dans les amortisseurs et les masses polaires. Il suffit dans ce cas soit d'augmenter la valeur de la résistance, soil de démarrer avec le circuit des inducteurs ouvert.

Selon la puissance et la tension des unités, l'appareillage de commande diffère, de mème que le processus des diverses manœuvres à effectuer pour la mise en route.

En général, pour les petites unités à basse tension, on emploie des aulo-transformateurs spéciaux semblables à ceux utilisés pour le démarrage des moteurs asynchrones à cage d'écureuil. Le circuil d'excitation reste fermé sur le rhéostat pendant toute la durée de la mise en vitesse, el en fin de démarrage, lorsque le moteur fonctionne sous tension normale à sa vitesse de synchronisme le dernier plot du contrôleur ouvre le neutre de l'autotransformateur. Ce dernier appareil qui n'est utilisé que pendant la période de démarrage proprement dite et mis hors circuit ensuite peut être établi pour un prix relativement faible en raison des surcharges momentanées qu'on peut lui faire supporter.

Pour des puissances supérieures on remplace l'auto-transformateur spécial de démarrage (qui n'est de construction normale que jusqu'à 150 ou $200 \mathrm{kVA}$ ) par un simple auto-transformateur à air. Dans ce cas la commande se fait par un inverseur et on a soin de ramener le neutre sur le tableau de commande, neutre qu'on ouvre ensuite avec un interrupteur bipolaire. Cette double manœuvre peut également se faire avec'un commutateur spécial.

Si le condensateur synchrone est alimenté par le secondaire d'un transformateur abaisseur, on sortira des prises supplémentaires sur ce dernier et on effectuera le démarrage comme dans le cas précédent, sans cependant toucher au neutre du transformateur.

Quelquefois, avec des grosses unités, on évite l'à coup de la première à la deuxième position en effectuant le passage phase par phase.

Dans le cas des condensateurs synchrones haute tension alimentés directement par le réseau on a recours également à un auto-transformateur, le changement de prises se fait à l'aide d'un combinateur spécial dans l'huile (comprenant un inverseur et un interrupteur bipolaire du neutre) que l'on place en série sur le disjoncteur automatique assurant la protection de la machine.

Dans tout les cas, le circuit d'excitation peut rester fermé sur lui-même pendant tout le démarrage.

Signalons un moyen assez employé de réduire les $\mathrm{kVA}$ absorbés lors du démarrage des grosses unités, qui consiste à injecter de l'huile sous pression dans les coussinets inférieurs pour faciliter le décollage; dans ce cas, il est nécessaiee de disposer d'une pompe à huile actionnée par un moteur indépendant.

\section{Construction des Condensateurs stenchrones}

La construction des condensateurs synchrones ne diffère pas dans ses grandes lignes de celle des alternateurs.

Les figures 6 et 8 représentent des condensateurs de faible puissance. Ces machines comportent des paliers boucliers fixés et centrés sur la carcasse, disposition qui permet de réaliser un encombrement minimum tout en assurant un bon centrage de la roue polaire. L'excitatrice montée en bout d'arbre est soutenue soit par trois colonnettes, soit par une chaise spéciale fixée sur le plateau bouclier.

Dans la figure 8 nous donnons une vue de condensateur de moyenne puissance avec paliers séparés montés sur socle et moteur auxiliaire de démarrage.

La figure 9 représente un condensateur de grande puissance prévu également avec moteur auxiliaire de démarrage.

Nous ne voulons point entrer dans le détail de la construction des condensateurs synchrones, nous insisterons plus particulièrement sur quelques différences importantes qui existent avec la construction des alternateurs.

Tout d'abord on réalise une économie sensible en adoptant la vitesse optima compatible avec la puissance apparente de la machine. Il en résulte que cette vitesse sera toujours plus grande que celle des alternateurs ou moteurs synchrones correspondants 
pour lesquels il y a lieu de tenir compte de la vilesse de la machine accouplée avec ces unités, On peut ainsi construire des condensateurs synchrones de grande puissance tout en conservant une carcasse en une seule pièce.

Ajoutons encore que le choix d'une vilesse élevée permet d'obtenir une ventilation excessivement énergique et qu'il n'est pas nécessaire mème pour des unités de puissance déjà considérable de recourir à une ventilation méthodique de la machine pour en assurer le refroidissement.

Toutefois cette vitesse élevée nécessite un équilibrage statique et dynamique parfait de la roue polaire.

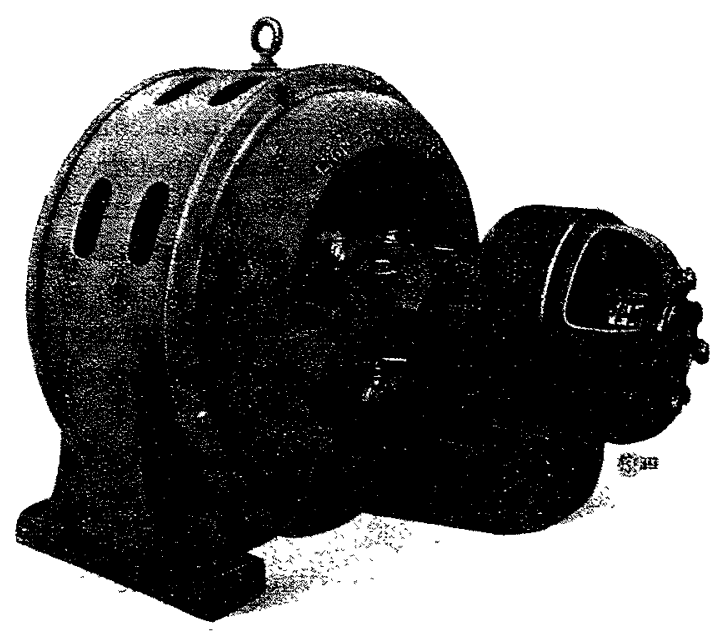

Fig. 8. - Condensateur $175 \mathrm{kVA}, 200 \mathrm{~V}, 750 \mathrm{l} / \mathrm{m}$ dimarrant en asynchrone.

Dans un condensateur synchrone qui ne fournit aucune puissance wattée autre que celle correspondant à ses pertes on peut alléger ses parties mécaniques, arbres, paliers, socle, etc., sans qu'il en résulte le moindre inconvénient. En outre il n'est point nécessaire de prévoir ces organes pour résister à une vitesse d'emballement qui, pour les alternateurs destinés à être accouplés à des turbines hydrauliques, atteint presque le double de la vitesse normale.

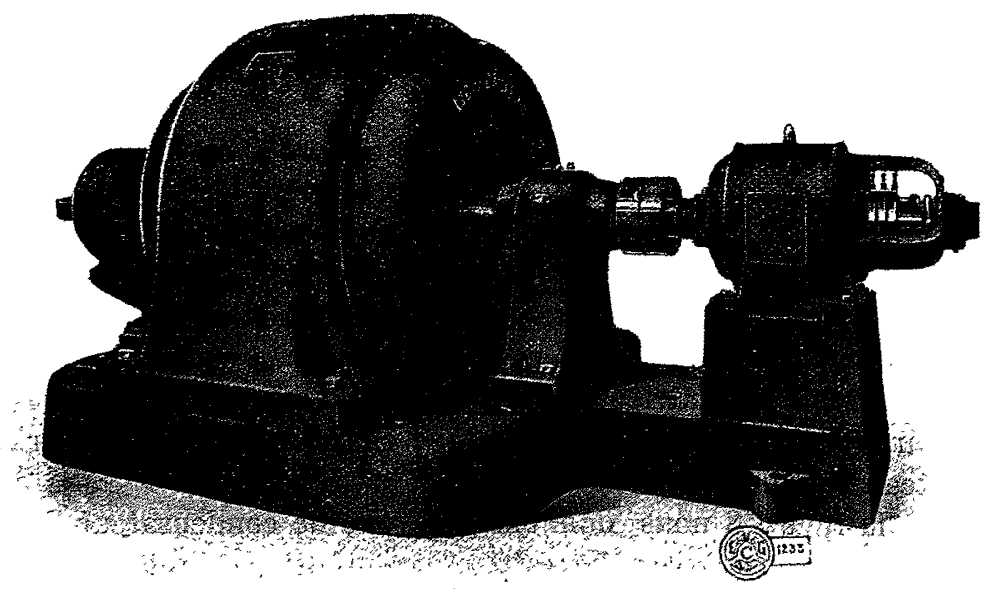

Fig. 9. - Condensateur synchrone $1.200 \mathrm{kVA}, 2.400 \mathrm{~V}, 1.000 \mathrm{t} / \mathrm{m}$, $50 \mathrm{pps}$.

Le seul point très particulier de la construction, et ceci aussi bien pour les moteurs synchrones que pour les condensateurs de moyennes et fortes puissances, consiste en l'adjonction de circuits amortisseurs sur la roue polaire.

Un condensateur synchrone doit être prévu pour pouvoir suivre les variations de fréquence du réseau sous peine de décrocher.
Pour de petites puissances les courants de Foucault développés dans les masses polaires par une variation relative de vitesse entre le champ tournant du stator et les inducteurs sont suffisants pour créer le couple synchronisant nécessaire.

Pour les puissances plus grandes, il est nécessaire de munir les pôles inducteurs d'amorlisseurs formant cage d'écureuil, fonctionnant comme un moteur asynchrone dans la période de perturbation et capable de créer un couple accélérateur ou retardateur en rapport avec l'inertie du rotor.

Ajoutons que celle cage d'écureuil doit être particulièrement bien étudiée dans le cas où le condensateur syuchrone doit démarrer en asynchrone.

Le bobinage de ces machines est exéculé comme celui des alternateurs : bobinage à fil tiré et encoches $1 / 2$ fermées pour les condensateurs de faible puissance à lension peu élevée, bobinage avec sections faites sur gabarits et compoundées avec encoches ouvertes pour les condensateurs de grande puissance à lension elevée. Signalons également que ces machines sont construites pour obtenir une $f . e$. m. parfaitement sinusoïdale, condition importante pour obtenir une marche trìs stable.

EMPLOI DL MOTEUR SYNCHRONE POUR LE RÉGLAGE DE LA TLASION

L'étude qui précède a été uniquement consacrée à l'amélioration du facteur de puissance par l'emploi d'un moteur synchrone fonctionnant comme condensateur. C'est là d'ailleurs l'applic'ation la plus courante.

Il y a cependant lieu de signaler que cette même machine peut produire une puissance réactive décalée en arrière et jouer ainsi le rôle de self. Cette propriété trouve notamment son application pour la mise sous tension des longues lignes de transmission. Ce problème est lié à celui de la régulation aux bornes des génératrices dont nous donnons ci-dessous un léger aperçu.

Nous examinerons le cas d'une centrale débitant sur une ligne:

$1^{\circ}$ Lorsque la charge est nulle ;

$2^{\circ}$ A $1 / 2$ charge.

$3^{\circ}$ A pleine charge.

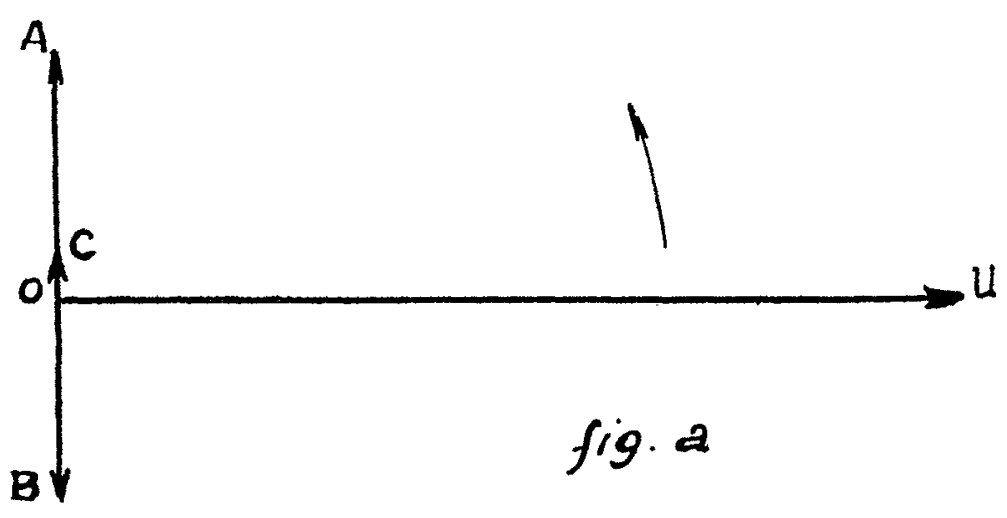

Dans le premier cas, l'intensilé fournie par les alternateurs cst entièrement déwattée en avant (fig. $a$ ) courant de capacité de la ligne dont l'effet bien connu est d'augmenter les ampères tours magnétisants. Il peut en résulter que même avec le minimum d'ampères tours d'excitation (rhéostat d'excitation au maximum) la tension fournie par les alternateurs peut dépasser notablement la tension normale et devenir de ce fait dangereux pour l'isolet ment.

L'emploi d'un moteur synchrone sous-excité permet de ramener le courant débité par les alternateurs à une valeur convenable pour permettre un bon réglage de la tension aux bornes. 
Dans le fonctionnement de la ligne à demi-charge (figure $b$ ) le couranl lourni par les alternateurs sera la résultante $O \mathrm{C}$ des vecteurs () A el O B. Suivant la grandeur respectuve de $O \mathrm{~A}$ et ()B el la valeur de $O$ l'intensité $O \mathrm{C}$ sera décalée en avant ou en arrière sur la tension d'un angle généralement faible. L'emploi du moteur synchrone fonctionnant soit comme self soit comme condensateur permettra facilement de ramener le facteur de puissance au départ à une valeur très voisine de l'unité .

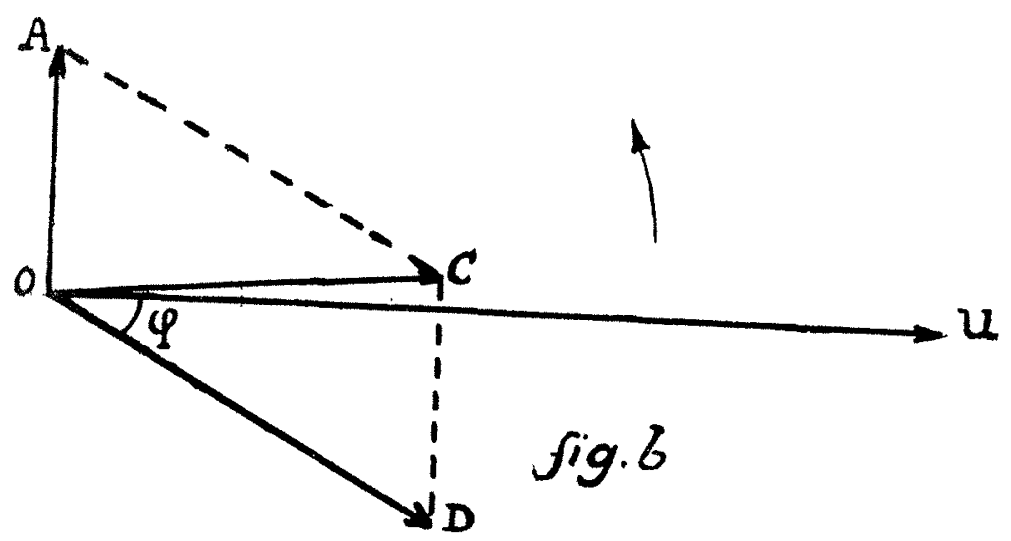

Enfin dans la marche à pleine charge (fig. c) le courant résultant () C fourni par les alternateurs sera généralement décalé en arrière. Les ampères tours démagnétisants qui résultent de ce fonctiomement devront ètre compensés par les ampères tours d'excitation supplémentaires pour obtenir la tension normale aux bornes de la machine. L'emploi du condensateur synchrone en permellant de relever le facteur de puissance au départ facilite le réglage de la lonsion.

En résumé, le fonctionnement d'alternateurs branchés sur une longue ligne de transmission peut nécessiter entre la marche $\therefore$ vide el la marche à pleine charge un réglage excessivement étendu de l'excitation de ces machines, incompatible souvent avec une construction normale ou un réglage automatique L'utilisation d'un moteur synchrone, par simple variation de son

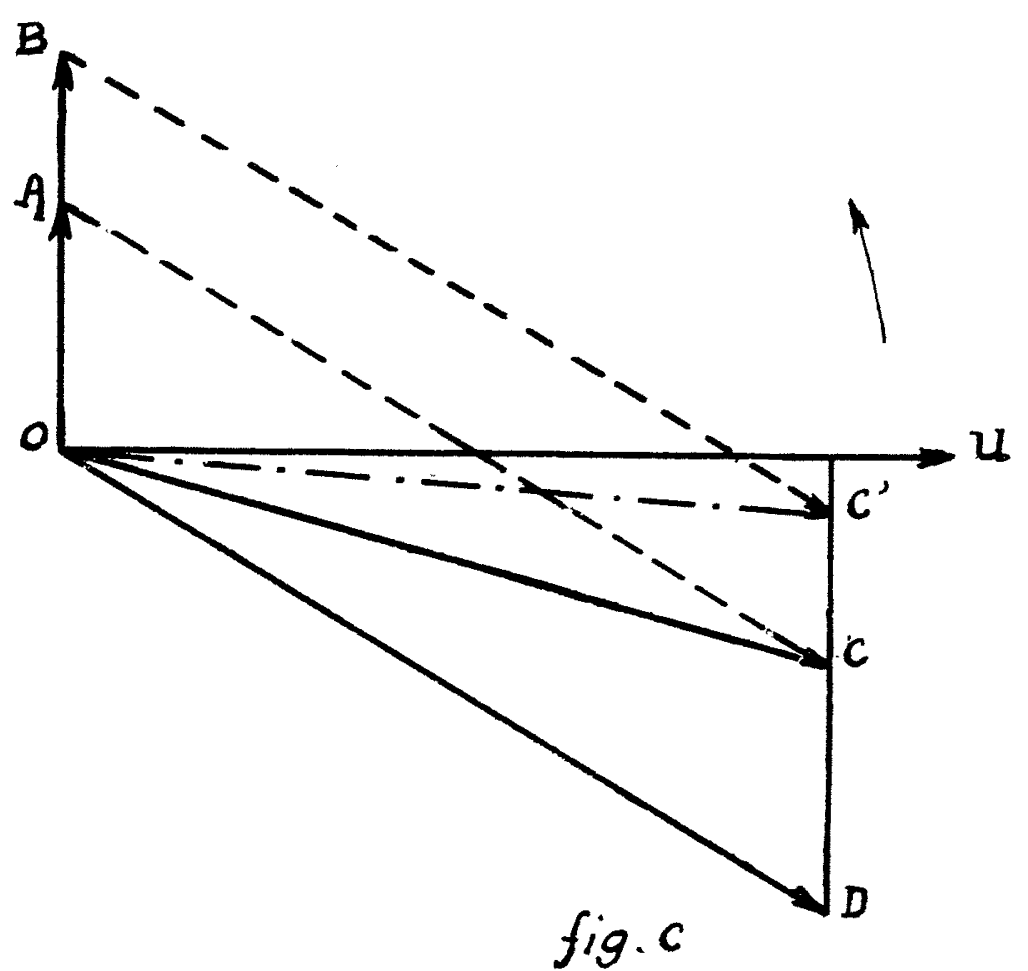

Fig. 10 .

excitation, permettra d'assurer dans de bonnes conditions la régulation de la tension. Toutes ces considérations sont nettement mises en évidence dans l'application que nous donnons ci-après.
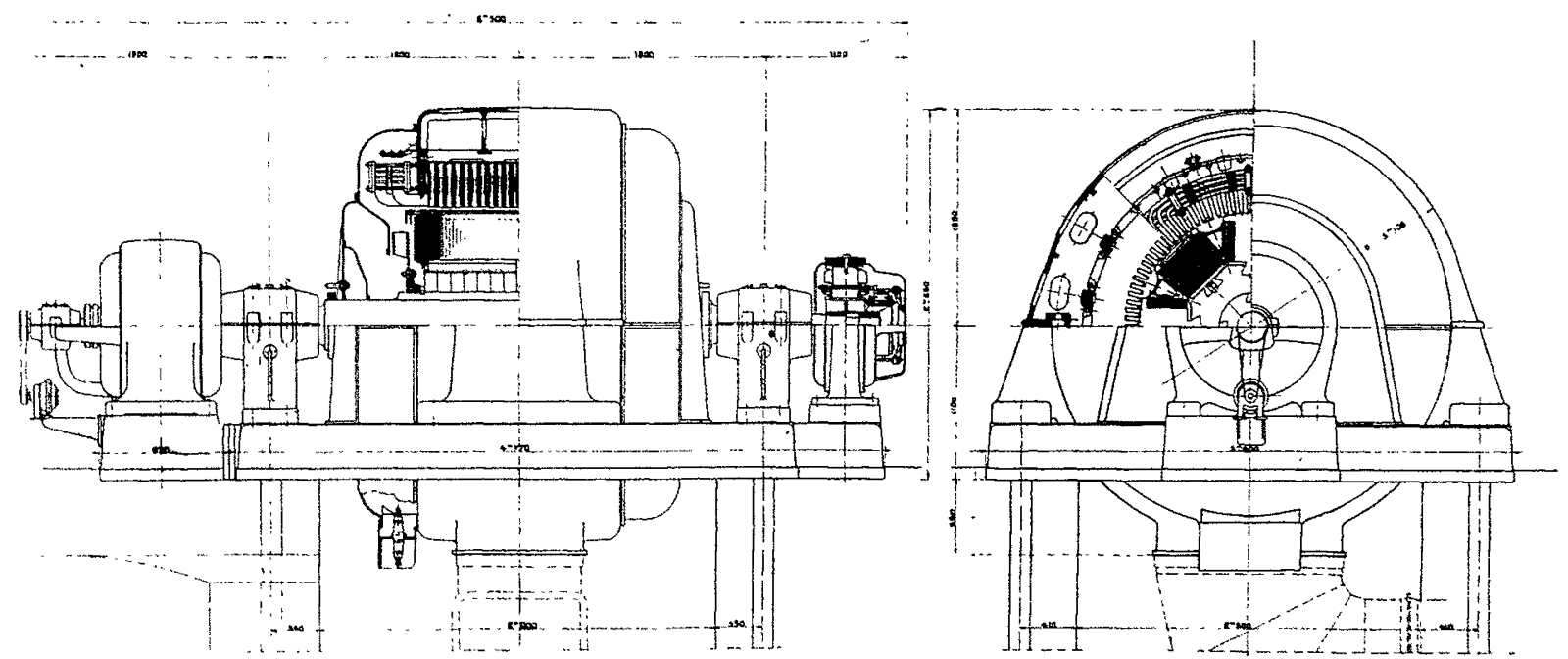

Fig. 11. - Plan d'ensemble d'un condensateur synchrone de $10.000 \mathrm{kVA}, 10.000 \mathrm{~V}, 750 \mathrm{t} / \mathrm{m}$.

QUELQUeS APPLICATIONS DES

Nous dounons ci-dessous à titre d'exemple deux cas intéressants d'application de ces machines.

Condensateur $1200 \mathrm{kVA}-2400$ Volts $-1000 \mathrm{t} / \mathrm{m}-50 \mathrm{pps}$

Ce condensateur était destiné à une grande Société métallurgique utilisant des moteurs de laminoir de grande puissance
Condensateurs synchrones

(600-1000 chevaux) et un nombre assez considérable de moteurs de puissances diverses, l'ensemble constituant une puissance moyenne absorbée de $3000 \mathrm{kVA}$ avec un cos assez faible 0,65 environ.

Le contrat avec le réseau de distribution comportait une pénalité croissante pour les facteurs de puissance inférieurs à 0,8 et une bonification lorsque ce dernier dépassait 0,85 . 
Afin de tenir compte des agrandissements possibles de l'usine, le condensateur synchrone devait permettre en l'état actuel de l'installation de ramener le facteur de puissance à une valeur comprise entre 0,85 et 0,90 . Il suffit de se rapporter aux graphiques de la fig. f pour voir que la puissance choisie de $1200 \mathrm{kVA}$ permet d'obtenir un cos ₹ de 0,87 et d'escompter ainsi une légère bonification. Il y avait encore lieu de tenir compte des variations continuelles de la charge et du facteur de puissance, qui peut ètre inférieur à 0,65 , lorsque, pars uite des circonstances, un grand nom- bre de moteurs marchent à faible charge ou à vide, absorbant cependant une puissance réactive sensiblement constante. On peut vérifier sur les graphiques précédents que, pour une puissance absorbée de $2400 \mathrm{KVA}$ avec $\cos \hat{\varphi}=0,5$, c'est-à-dire une puissance wallée de $1200 \mathrm{Kw}$ au lieu de la moyenne de 2000 envisagée précédemment, le condensateur synchrone permet encore d'obtenir un cos p égal à 0,8 .

Le lecteur trouvera ci-dessous quelques résultats d'essais sur ce condensateur,

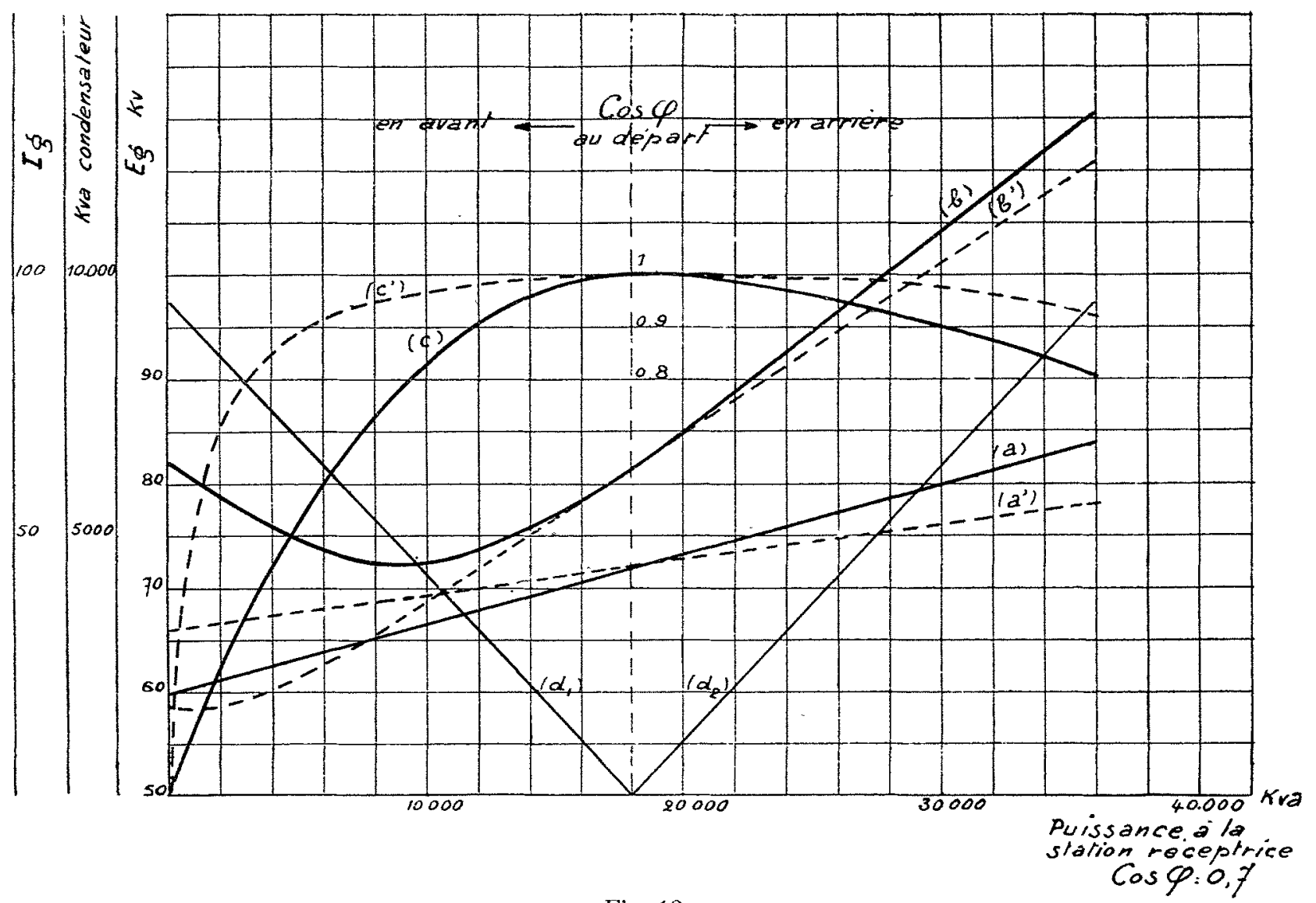

Fig. 12.

Condensateur $.10 .000 \mathrm{kVA}-10.000$ volts $-750 \mathrm{t} / \mathrm{m}-50 \mathrm{pps}$

Ce condensateur était destiné à fonctionner au bout d'une ligne de $350 \mathrm{~km}$. avec une tension composée de $110.000 \mathrm{~V}$ qui devait être maintenue constante quelle que soit la charge.

Le courant de capacité de la ligne de 62 ampères soit une puissance déwattée en avant de $11.800 \mathrm{kVA}$ imposait pour la mise sous tension de faire débiter au condensateur une puissance déwattée en arrière suffisante pour permettre un réglage normal de l'excitation des alternateurs.

Nous donnons, dans le tableau ci-dessous, les principaux résultats prévus avec le fonctionnement de ce condensateur pour une charge de la ligne variant de 0 à $36.000 \mathrm{kVA}$ avec un cos ? à la station réceptrice de 0,7 .
Les courbes $a, b$ et $c$ représentent respectivement en fonction de la charge les variations de la tension, de l'intensité et du facteur de puissance à la station génératrice due seulement à la ligne de transport. Les courbes $a^{\prime}, b^{\prime}, c^{\prime}$ représentent ces mêmes variations en utilisant un condensateur synchrone en bout de lignè dont la puissance déwattée utilisée est représentée par les droites $d_{1}$ (en arrière) et $d_{2}$ en avant.

Notons que la variation de tension, au départ, passe de $40 \%$ dans le premier cas à $17 \%$ dans la marche avec condensateur, valeur admissible pour pouvoir obtenir une bonne régulation. Cela permet d'utiliser pour ces transports d'énergie à grande distance des transformateurs à réactance élevée offrant une plus grande sécurité tout en améliorant légèrement le rendement de la ligne. 